\title{
A meta-analytic evaluation of cholesteryl ester transfer protein (CETP) C-629A polymorphism in association with coronary heart disease risk and lipid changes
}

\author{
Shouwei Lin ${ }^{1}$, Ruozhu Dai ${ }^{1}$, Rong Lin ${ }^{1}$ \\ ${ }^{1}$ Department of Cardiology, Fujian Medical University Affiliated First Quanzhou Hospital, Fujian Province, P.R. China \\ Correspondence to: Shouwei Lin, email: linshouwei2016@126.com \\ Keywords: coronary heart disease, cholesteryl ester transfer protein, polymorphism, association, meta-analysis \\ Received: August 15, $2016 \quad$ Accepted: October 19, $2016 \quad$ Published: October 25, 2016
}

\section{ABSTRACT}

Lipid metabolism plays an essential role in the pathogenesis of atherosclerosis, a major cause for coronary heart disease (CHD). Cholesteryl ester transfer protein (CETP) is an important glycoprotein involved in lipid metabolism by transferring cholesteryl esters to apolipoprotein B-containing lipoproteins in exchange for triglycerides. The objective of this meta-analysis was to evaluate the association of CETP C-629A polymorphism with CHD risk and lipid changes. Four public databases were searched, and data from 17 qualified articles were extracted in duplicate and analyzed by STATA software. Overall association of C-629A with CHD risk was nonsignificant in 5441 patients and 7967 controls. Subgroup analyses by ethnicity revealed significance only in Caucasians, with the odds of CHD being 1.18, 1.43 and 1.41 under allelic, genotypic and dominant models, respectively $(P<0.001)$. Similarly, the $-629 \mathrm{C}$ allele increased the corresponding risk of myocardial infarction by 1.23-, 1.28- and 1.29-fold $(P<0.02)$. The association of $C-629 A$ with $C H D$ was significantly strengthened in prospective and large studies. Moreover, carriers of the $-629 \mathrm{C}$ allele had significant higher levels of circulating CETP (weighted mean difference [WMD]: $0.45 \mu \mathrm{g} / \mathrm{mL} ; 95 \%$ confidence interval [CI]: 0.25 to $0.65 ; P<0.001$ ), but lower levels of high-density lipoprotein cholesterol (HDL-C) (WMD: $-\mathbf{3 . 6 5} \mathbf{m g} / \mathrm{dL}$; $95 \% \mathrm{CI}$ : $\mathbf{- 5 . 5 9}$ to $-1.70 ; P<0.001$ ) relative to the $-629 \mathrm{AA}$ homozygotes. The probability of publication bias was low. Our meta-analytic findings collectively demonstrate that the $-629 \mathrm{C}$ allele was significantly associated with an increased risk of CHD in Caucasians, and this association may be mediated by its phenotypic regulation on circulating CETP and HDL-C.

\section{INTRODUCTION}

It is widely accepted that lipid metabolism plays an essential role in the pathogenesis of atherosclerosis, a major cause for coronary heart disease (CHD) $[1,2]$. The past decade has witnessed substantial advances in understanding the genetic basis of lipid abnormalities of biomedical importance. In particular, cholesteryl ester transfer protein (CETP) is a plasma glycoprotein involved in lipid metabolism, and it can trigger the transfer of cholesteryl esters from high-density lipoprotein (HDL) to apolipoprotein B-containing lipoproteins in exchange for triglycerides, a key step known as 'reverse cholesterol transport' [3]. The gene encoding CETP is shipped with 2056 polymorphic loci (https://www.ncbi. nlm.nih.gov/gene/1071), and some of them have been proposed as potential regulators of CETP deficiency and HDL cholesterol (HDL-C) increase, as indicated by a comprehensive meta-analysis of 92 published studies [4]. However, the exact mechanism whereby CETP genetic loci alter susceptibility to CHD remains largely unknown. A clear understanding of how CETP genetic loci regulate lipid metabolism associated with CHD is therefore a challengeable task. To take a step further, we in this study meta-analytically evaluated the association of a promoter functional polymorphism, C-629A (rs1800775) in CETP with CHD risk and lipid changes. This polymorphism was reported to be a Sp1/Sp3 transcription factor binding site that can regulate the transcriptional activity of human CETP promoter $[5,6]$. 


\section{RESULTS}

\section{Eligible articles}

Figure 1 is a flow diagram that depicted the steps of filtrating articles for this meta-analysis. From 459 initially identified articles from 4 electronic databases, 17 that satisfied our eligibility criteria were finally analyzed [7-24]. There were 12 qualified articles including 16 study groups (5441 CHD patients and 7967 controls) for the association between CETP C-629A polymorphism and CHD risk [7-18]. There were 10 qualified articles including 20 study groups (22488 subjects) for the relationship between CETP C-629A polymorphism and circulating lipid changes [13, 15-17, 19-24].

\section{Study characteristics}

Table 1 (A, B, C) summarizes the characteristics of all study groups, and Supplementary Table S1 provides the mean values of lipid concentrations under study across C-629A genotypes. For the genotype-disease association, 7 of 16 study groups were based on East Asians, 3 on Caucasians, 2 on Middle Easterns and 4 on mixed populations. Coronary stenosis was assessed in 9 study groups and myocardial infarction in 7 study groups. Ten study groups enrolled controls from general populations and 6 from hospitals. Twelve studies were in retrospective designs and 4 in prospective designs. Age was reported to be matched between CHD patients and controls by 11 studies (Table 1).

CHD patients were slightly older than controls (mean age: 56.74 vs. 52.99 years, $P=0.053$ ) and gender composition was comparable $(P=0.111)$. Mean levels of BMI $(P=0.005)$, smoking status $(P<0.001)$, hypertension $(P=0.001)$ and diabetes $(P=0.182)$ were significantly higher in CHD patients than in controls. By contrast, controls had significant higher levels of circulating HDL-C $(P=0.001)$ and Apo-AI $(P=0.026)$ than patients.

For the genotype-phenotype relationship, circulating HDL-C was investigated in 16 study groups and triglycerides in 9 groups, LDL-C in 9 groups, CETP in 4 groups, Apo-AI and Apo-B respectively in 3 groups, as shown in Supplementary Table S1.

\section{CETP C-629A polymorphism and CHD risk}

Table 2 shows the overall and subgroup analyses of CETP C-629A polymorphism in association with CHD risk. In overall analyses, the $-629 \mathrm{C}$ allele was nonsignificantly associated with a $4 \%(95 \% \mathrm{CI}: 0.95$ to $1.15 ; P=0.412)$, $15 \%$ (95\% CI: 0.98 to $1.35 ; P=0.090)$ and $14 \%(95 \% \mathrm{CI}$ : 0.99 to $1.31 ; P=0.081)$ increased risk under allelic $(-629 \mathrm{C}$ allele versus -629A allele), homozygous genotypic (-629CC genotype versus -629AA genotype) and dominant (-629CC genotype plus -629AC genotype versus -629AA genotype) models, respectively. These associations were obsessed by moderate heterogeneity, with the corresponding $P^{2}$ statistic of being $69.1 \%, 50.2 \%$ and $60.4 \%$. There was a low probability of publication bias except for dominant model (Egger's test: $P=0.055$ ) (Figure 2). Additionally, in 5 studies involving only males, effect estimates were slightly reinforced relative to the overall estimates, and significance was detected under dominant model $(\mathrm{OR}=1.22 ; 95 \% \mathrm{CI}: 1.02$ to $1.47 ; P=$ $0.033)$ with moderate heterogeneity $\left(I^{2}=53.0 \%\right)$.

Stratifying study groups by ethnicity identified significance only in Caucasians, with the odds of CHD being $1.18,1.43$ and 1.41 respectively under allelic, homozygous genotypic and dominant models $(P<0.001$ for all), without observable heterogeneity $\left(I^{2}=0 \%\right.$ for all). In contrast, the effect estimates were in an opposite direction, albeit nonsignificant in Middle Easterns across three genetic models.

In subgroup analyses by CHD subtypes, the $-629 \mathrm{C}$ allele was observed to significantly increase risk of myocardial infarction by 1.23-, 1.28- and 1.29-fold respectively under allelic $(P=0.015)$, homozygous genotypic $(P=0.015)$ and dominant $(P=0.001)$ models, with borderline heterogeneity. By source of controls, the effect estimates were roughly comparable between hospital- and population-based studies, with significant heterogeneity.

By study design, the $-629 \mathrm{C}$ allele seemed to confer a $21 \%$ to $28 \%$ increased risk for CHD in prospective studies across three genetic models $(P<0.05)$ without evident heterogeneity, while this risk was reduced towards the unity in retrospective studies.

When the analysis was restricted to the large study ( $\geq 500$ subjects), pooled risk estimates were statistically significant under allelic ( $\mathrm{OR}=1.12 ; P=0.004)$, homozygous genotypic $(\mathrm{OR}=1.25 ; P=0.005)$ and dominant $(\mathrm{OR}=1.25$; $P=0.001$ ) models with borderline heterogeneity, while an opposite yet nonsignificant association was identified in the small studies $(<500$ subjects $)$.

\section{CETP C-629A polymorphism and lipid changes}

Table 3 presents the overall analyses of CETP C-629A polymorphism with circulating lipid changes under both homozygous genotypic and dominant models. An increase in circulating CETP was observed for carriers of the $-629 \mathrm{CC}$ genotype (WMD: $0.70 \mu \mathrm{g} / \mathrm{mL} ; 95 \% \mathrm{CI}: 0.30$ to $1.10 ; P=0.001$ ) or $-629 \mathrm{C}$ allele (WMD: $0.45 \mu \mathrm{g} / \mathrm{mL}$; $95 \%$ CI: 0.25 to $0.65 ; P<0.001)$ relative to the $-629 \mathrm{AA}$ homozygotes, with evident heterogeneity. By contrast, there was a reduced yet nonsignificant trend in circulating triglycerides for -629CC genotype or -629C allele carriers, and the probabilities of heterogeneity and publication bias were low.

Circulating HDL-C was significantly reduced in carriers of the $-629 \mathrm{CC}$ genotype (WMD: $-4.36 \mathrm{mg} / \mathrm{dL}$; 95\% CI: -7.20 to $-1.51 ; P=0.003$ ) or $-629 \mathrm{C}$ allele (WMD: $-3.65 \mathrm{mg} / \mathrm{dL} ; 95 \%$ CI: -5.59 to $-1.70 ; P<0.001)$ when compared with the -629AA homozygotes, with moderate 
heterogeneity and a low probability of publication bias. The -629CC genotype or -629C allele was associated with higher circulating LDL-C than the -629AA genotype, while no significance was reached. Similarly, the -629CC genotype or -629C allele was associated with lower ApoAI but higher Apo-B than the -629AA genotype, with no observable heterogeneity.

\section{Meta-regression analyses}

To further seek possible causes of clinical heterogeneity, meta-regression analyses that modeled age, male gender, BMI, smoking, dyslipidemia, hypertension, diabetes, circulating triglycerides, total cholesterol, HDL-C, LDL-C, CETP, Apo-AI and Apo-B if available under study were conducted, and none of these factors contributed significantly to the association of CETP C-629A polymorphism with CHD risk (all $P>0.05$ ).

\section{Cumulative and influential analyses}

Cumulative analyses by ascending publication years indicated no substantive change in the direction of effect estimates with the addition of subsequent studies (Supplementary Figure S1). In addition, influential analyses confirmed the stability of overall effect estimates (Supplementary Figure S2).

\section{DISCUSSION}

The objective of this meta-analysis was to evaluate the association of CETP C-629A polymorphism with the risk of CHD and lipid changes by summarizing data from 17 articles. The key finding of this study was that the $-629 \mathrm{C}$ allele was significantly associated with an increased risk of CHD in Caucasians, and this association may be mediated by its phenotypic regulation on circulating CETP and HDL-C.

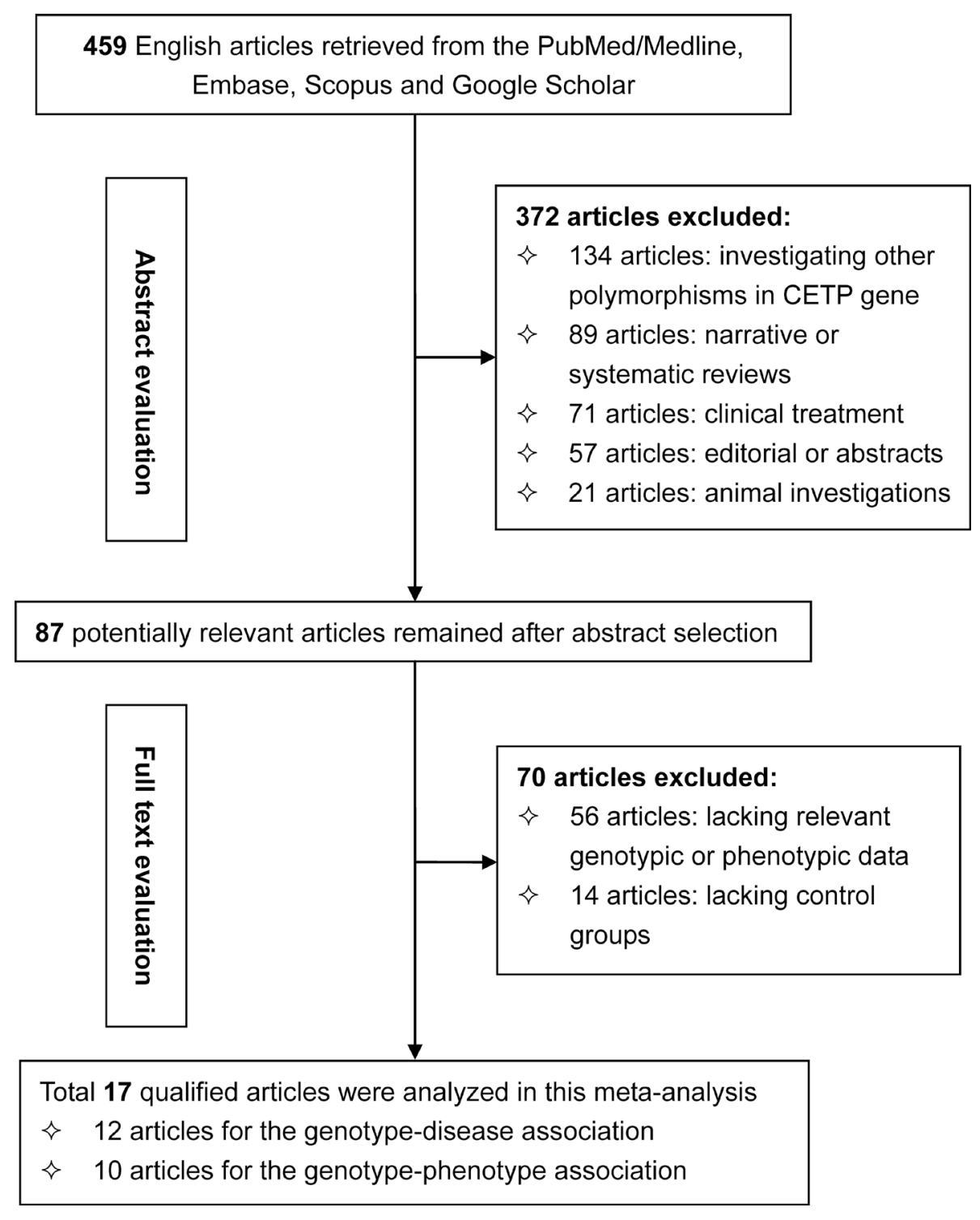

Figure 1: Flow diagram depicting the steps of article selection for this meta-analysis. 
Table 1A: The baseline characteristics of all eligible articles for the genotype-disease association

\begin{tabular}{|c|c|c|c|c|c|c|c|c|}
\hline Author (year) & Ethnicity & CHD subtype & Source & Design & Matched & Genotyping & Patients & Controls \\
\hline Eiriksdottir G (2001) & Caucasian & Myocardial infarction & Population & Prospective & NR & RFLP & 388 & 794 \\
\hline Freeman DJ (2003) & Caucasian & Myocardial infarction & Population & Prospective & YES & Non-RFLP & 498 & 1108 \\
\hline Tobin MD (2004) & Caucasian & Myocardial infarction & Hospital & Retrospective & NA & Non-RFLP & 547 & 505 \\
\hline Zheng K (2005) & Asian & Coronary stenosis & Hospital & Retrospective & YES & Non-RFLP & 203 & 209 \\
\hline Zee RY (UK) (2006) & Mixed & Myocardial infarction & Population & Prospective & YES & Non-RFLP & 547 & 505 \\
\hline Zee RY (PHS) (2006) & Mixed & Myocardial infarction & Population & Prospective & YES & Non-RFLP & 523 & 2092 \\
\hline Meiner V (males) (2008) & Mixed & Myocardial infarction & Population & Retrospective & YES & Non-RFLP & 321 & 308 \\
\hline Meiner V (females) (2008) & Mixed & Myocardial infarction & Population & Retrospective & YES & Non-RFLP & 256 & 351 \\
\hline Tanrikulu S (2009) & Middle Eastern & Coronary stenosis & Hospital & Retrospective & YES & RFLP & 120 & 120 \\
\hline Poduri A (2009) & Asian & Coronary stenosis & Population & Retrospective & YES & RFLP & 265 & 150 \\
\hline Padmaja N (2009) & Asian & Coronary stenosis & Hospital & Retrospective & YES & RFLP & 504 & 338 \\
\hline Ghatreh Samani K et al (2009) & Middle Eastern & Coronary stenosis & Hospital & Retrospective & YES & RFLP & 187 & 136 \\
\hline Wang J (2013) & Asian & Coronary stenosis & Hospital & Retrospective & YES & Non-RFLP & 420 & 424 \\
\hline Lu Y (Chinese) (2013) & Asian & Coronary stenosis & Population & Retrospective & NR & RFLP & 442 & 383 \\
\hline Lu Y (Malays) (2013) & Asian & Coronary stenosis & Population & Retrospective & NR & RFLP & 110 & 155 \\
\hline Lu Y (Indian) (2013) & Asian & Coronary stenosis & Population & Retrospective & NR & RFLP & 110 & 389 \\
\hline
\end{tabular}

Notes: CHD, coronary heart disease; NR, not reported; RFLP, restriction fragment length polymorphism; BMI, body mass index; TG, triglycerides; TC, total cholesterol; HDL-C and LDL-C, high- and low-density lipoprotein cholesterol; CETP, cholesteryl ester transfer protein; Apo-AI, apolipoprotein AI; Apo-B, apolipoprotein B.

Table 1B: The demographic characteristics of all study populations for the genotype-disease association

\begin{tabular}{|c|c|c|c|c|c|c|c|c|c|c|c|c|c|}
\hline \multicolumn{2}{|c|}{ Age (years) } & \multicolumn{2}{|c|}{ Gender } & \multicolumn{2}{|c|}{ BMI $\left(\mathbf{k g} / \mathbf{m}^{2}\right)$} & \multicolumn{2}{|c|}{ Smoking } & \multicolumn{2}{|c|}{ Dyslipidemia } & \multicolumn{2}{|c|}{ Hypertension } & \multicolumn{2}{|c|}{ Diabetes } \\
\hline Patients & Controls & Patients & Controls & Patients & Controls & Patients & Controls & Patients & Controls & Patients & Controls & Patients & Controls \\
\hline 71.0 & 76.0 & 1.000 & 1.000 & 27.30 & 26.00 & NR & NR & NR & NR & NR & NR & NR & NR \\
\hline 56.9 & 56.7 & 1.000 & 1.000 & 26.00 & 25.60 & 0.530 & 0.550 & NR & NR & NR & NR & NR & NR \\
\hline 61.9 & 58.6 & 0.680 & 0.620 & 25.90 & 25.70 & 0.402 & 0.170 & NR & NR & 0.310 & 0.168 & 0.087 & 0.020 \\
\hline 55.4 & 54.8 & 0.675 & 0.675 & 25.92 & 23.89 & 0.680 & 0.411 & NR & NR & 0.552 & 0.167 & NR & NR \\
\hline 58.3 & 58.4 & 1.000 & 1.000 & 25.50 & 25.00 & 0.571 & 0.566 & 0.132 & 0.083 & NR & NR & 0.056 & 0.027 \\
\hline 58.3 & 58.4 & 1.000 & 1.000 & 25.50 & 25.00 & 0.571 & 0.566 & 0.132 & 0.083 & NR & NR & 0.056 & 0.027 \\
\hline 44.0 & 42.2 & 1.000 & 1.000 & 29.00 & 26.60 & 0.468 & 0.203 & 0.449 & 0.250 & 0.375 & 0.172 & 0.108 & 0.020 \\
\hline 50.5 & 49.5 & 0.000 & 0.000 & 29.70 & 26.90 & 0.537 & 0.128 & 0.420 & 0.282 & 0.450 & 0.239 & 0.220 & 0.048 \\
\hline 54.0 & 52.0 & 0.780 & 0.483 & 32.00 & 25.00 & 0.558 & 0.225 & NR & NR & 0.467 & 0.108 & NR & NR \\
\hline 47.5 & 47.0 & 0.838 & 0.760 & 28.18 & 23.53 & 0.351 & 0.200 & NR & NR & NR & NR & NR & NR \\
\hline 50.7 & 49.7 & 0.909 & 0.888 & 24.08 & 23.61 & 0.423 & 0.257 & NR & NR & 0.381 & 0.305 & 0.337 & NR \\
\hline 54.6 & 52.8 & NR & NR & 27.10 & 26.90 & NR & NR & NR & NR & NR & NR & NR & NR \\
\hline 66.0 & 66.0 & 0.398 & 0.396 & 24.30 & 24.20 & 0.510 & 0.323 & NR & NR & 0.488 & 0.387 & 0.210 & 0.120 \\
\hline 59.3 & 42.7 & 0.781 & 0.539 & 24.24 & 23.00 & 0.529 & 0.174 & 0.326 & 0.463 & 0.691 & 0.082 & 0.433 & 0.027 \\
\hline 59.1 & 40.7 & 0.761 & 0.913 & 26.14 & 25.17 & 0.490 & 0.527 & 0.275 & 0.564 & 0.727 & 0.041 & 0.626 & 0.034 \\
\hline 60.4 & 42.4 & 0.835 & 0.622 & 24.90 & 24.77 & 0.438 & 0.136 & 0.211 & 0.643 & 0.618 & 0.098 & 0.618 & 0.064 \\
\hline
\end{tabular}


Table 1C: The circulating lipid profiles of all study populations for the genotype-disease association

\begin{tabular}{|c|c|c|c|c|c|c|c|c|c|c|c|c|c|}
\hline \multicolumn{2}{|c|}{ TG (mg/dL) } & \multicolumn{2}{|c|}{$\mathrm{TC}(\mathrm{mg} / \mathrm{dL})$} & \multicolumn{2}{|c|}{ HDL-C (mg/dL) } & \multicolumn{2}{|c|}{ LDL-C (mg/dL) } & \multicolumn{2}{|c|}{$\operatorname{CETP}(\mu \mathrm{g} / \mathrm{mL})$} & \multicolumn{2}{|c|}{ Apo-AI (mg/dL) } & \multicolumn{2}{|c|}{ Apo-B (mg/dL) } \\
\hline Patients & Controls & Patients & Controls & Patients & Controls & Patients & Controls & Patients & Controls & Patients & Controls & Patients & Controls \\
\hline 101.86 & 94.77 & 232.02 & 228.15 & 44.08 & 43.70 & NR & NR & NR & NR & NR & NR & NR & NR \\
\hline 173.61 & 162.98 & 273.78 & 271.46 & 41.38 & 44.08 & 194.12 & 191.42 & NR & NR & NR & NR & NR & NR \\
\hline NR & NR & NR & NR & NR & NR & NR & NR & NR & NR & NR & NR & NR & NR \\
\hline 142.60 & 120.46 & 193.74 & 189.48 & 45.24 & 50.66 & 95.13 & 84.69 & NR & NR & 119.00 & 125.00 & 111.00 & 107.00 \\
\hline NR & NR & NR & NR & NR & NR & NR & NR & NR & NR & NR & NR & NR & NR \\
\hline NR & NR & NR & NR & NR & NR & NR & NR & NR & NR & NR & NR & NR & NR \\
\hline 247.40 & 197.50 & NR & NR & 38.20 & 43.30 & NR & NR & NR & NR & NR & NR & NR & NR \\
\hline 217.90 & 160.50 & NR & NR & 47.40 & 59.40 & NR & NR & NR & NR & NR & NR & NR & NR \\
\hline 164.00 & 136.00 & 201.00 & 204.00 & 38.00 & 47.00 & 131.00 & 129.00 & NR & NR & NR & NR & NR & NR \\
\hline 189.25 & 138.81 & 203.55 & 147.22 & 35.51 & 41.15 & 130.18 & 78.31 & NR & NR & NR & NR & NR & NR \\
\hline 147.10 & 120.30 & 195.70 & 169.04 & 40.90 & 40.62 & 122.90 & 115.92 & NR & NR & NR & NR & NR & NR \\
\hline 190.90 & 184.90 & 176.60 & 172.60 & 36.70 & 38.90 & 101.60 & 96.70 & 1.98 & 2.31 & 120.50 & 125.90 & 104.30 & 102.70 \\
\hline NR & NR & 182.52 & 158.16 & 46.40 & 47.56 & 109.44 & 94.74 & NR & NR & NR & NR & NR & NR \\
\hline 121.35 & 162.98 & 176.72 & 224.67 & 37.51 & 54.14 & 107.50 & 138.05 & NR & NR & 119.47 & 144.40 & 89.78 & 106.20 \\
\hline 133.75 & 185.12 & 177.88 & 226.22 & 35.19 & 46.02 & 111.76 & 143.08 & NR & NR & 115.29 & 129.29 & 103.64 & 121.36 \\
\hline 114.26 & 170.95 & 164.35 & 216.94 & 34.03 & 42.92 & 99.77 & 139.99 & NR & NR & 110.01 & 135.85 & 102.30 & 122.87 \\
\hline
\end{tabular}

The importance of the current study lies in deepening our understanding of the functional aspects of CETP genetic variation involved in the pathogenesis of CHD.

Lately, a large comprehensive meta-analysis choosing CETP gene TaqIB (rs708272) polymorphism as an instrument has demonstrated that circulating CETP may play a causal role in the pathophysiology of CHD [25], although there are still some unresolved issues revolving around the prerequisites of Mendelian randomization analysis [26], such as pleiotropic impact of genetic polymorphism under study and linkage disequilibrium with another locus that differently modifies circulating CETP. Nevertheless, it still remains an open question to interrogate CETP genetic loci associated with CHD risk and responsible for the changes of biologically relevant lipids. The conduct of this meta-analysis therefore represents a supplement to medical research and deepens our understanding of the genetics of CHD.

As indicated in this meta-analysis, CETP C-629A mutation can alter susceptibility to CHD in Caucasian populations, at least in part, through its phenotypic regulation on circulating CETP and HDL-C. Several cautionary notes regarding the interpretation and extrapolation of this finding should be sounded. First, genetic heterogeneity across ethnicities is a common phenomenon gripping a majority of association studies. It is of interest to found that CETP $-629 \mathrm{C}$ allele was significantly associated with an increased risk of CHD only in Caucasians, while this association was reversed to be protective in Asian and Middle Eastern populations. As a matter of fact, linkage disequilibrium patterns are generally believed to be diverse across races or ethnicities. For example, the linkage of a genetic variant with another functional variant was usually strong in one ethnic group but weak or nonexistent in another [27]. Second, experimental data suggested the close association of CETP genetic alterations with increased large cholesterolenriched HDL particles [28]. Moreover, the fact that simple measurement of circulating HDL-C may not always reflect the potential cardioprotective activity of HDL particle, which might be dysfunctional in spite of high HDL-C can by no means be ignored [29]. It is widely recognized that cholesterol-overloaded HDL particle can not only decrease the hepatic selective uptake of cholesterol from HDL particle, but also exert a defective effect on efflux potential of cholesterol from extra-hepatic cells [30-32]. In addition, some pharmacological agents such as CETP inhibitors [33] and Niacin [34] that raise circulating HDL-C simultaneously increased the levels of cholesterol-overloaded particles. Third, the association of CETP C-629A polymorphism with CHD risk had a biological basis, as this polymorphism also accounted for the changes of circulating CETP concentrations. In addition, this association was strengthened after restricting analysis to the prospective and large studies, which further verifies the robustness of our meta-analytic findings.

A number of possible limitations should be recognized for this meta-analysis. First, only summary 
Table 2: Overall and subgroup analyses of CETP gene C-629A in susceptibility to CHD under three genetic models

\begin{tabular}{|c|c|c|c|c|c|c|c|c|c|c|c|c|c|}
\hline \multirow{2}{*}{ Groups } & \multirow{2}{*}{ Studies } & \multicolumn{4}{|c|}{ Allelic model } & \multicolumn{4}{|c|}{ Homozygous genotypic model } & \multicolumn{4}{|c|}{ Dominant model } \\
\hline & & OR & $95 \% \mathrm{CI}$ & $P$ & $I^{2}(\%)$ & OR & $95 \% \mathrm{CI}$ & $P$ & $I^{2}(\%)$ & OR & $95 \% \mathrm{CI}$ & $P$ & $I^{2}(\%)$ \\
\hline Overall & 16 & 1.04 & 0.95 to 1.15 & 0.412 & 69.1 & 1.15 & 0.98 to 1.35 & 0.090 & 50.2 & 1.14 & 0.99 to 1.31 & 0.081 & 60.4 \\
\hline Males only & 5 & 1.09 & 0.97 to 1.21 & 0.153 & 55.2 & 1.19 & 0.94 to 1.50 & 0.156 & 57.8 & 1.22 & 1.02 to 1.47 & 0.033 & 53.0 \\
\hline \multicolumn{14}{|l|}{ Ethnicity } \\
\hline Asian & 7 & 0.96 & 0.78 to 1.18 & 0.707 & 78.1 & 1.00 & 0.74 to 1.34 & 0.976 & 53.5 & 1.04 & 0.82 to 1.32 & 0.753 & 54.4 \\
\hline Caucasian & 3 & 1.18 & 1.08 to 1.30 & $<0.001$ & 0.0 & 1.43 & 1.18 to 1.74 & $<0.001$ & 0.0 & 1.41 & 1.20 to 1.66 & $<0.001$ & 0.0 \\
\hline Mixed & 4 & 1.08 & 0.92 to 1.27 & 0.363 & 67.8 & 1.17 & 0.83 to 1.64 & 0.374 & 68.6 & 1.21 & 0.93 to 1.56 & 0.153 & 64.8 \\
\hline Middle Eastern & 2 & 0.89 & 0.55 to 1.44 & 0.644 & 73.2 & 0.96 & 0.52 to 1.78 & 0.901 & 34.2 & 0.79 & 0.40 to 1.52 & 0.474 & 72.1 \\
\hline \multicolumn{14}{|l|}{ CHD subtypes } \\
\hline Coronary stenosis & 9 & 0.95 & 0.79 to 1.14 & 0.562 & 75.2 & 1.00 & 0.77 to 1.28 & 0.976 & 45.0 & 0.97 & 0.76 to 1.22 & 0.776 & 61.7 \\
\hline Myocardial infarction & 7 & 1.23 & 1.02 to 1.24 & 0.015 & 50.9 & 1.28 & 1.05 to 1.56 & 0.015 & 52.9 & 1.29 & 1.11 to 1.51 & 0.001 & 47.8 \\
\hline \multicolumn{14}{|l|}{ Source of controls } \\
\hline Hospital & 6 & 1.09 & 0.94 to 1.26 & 0.267 & 55.1 & 1.27 & 1.04 to 1.54 & 0.017 & 3.5 & 1.13 & 0.87 to 1.48 & 0.363 & 68.5 \\
\hline Population & 10 & 1.02 & 0.89 to 1.16 & 0.790 & 75.1 & 1.10 & 0.88 to 1.37 & 0.413 & 62.6 & 1.13 & 0.95 to 1.35 & 0.166 & 58.5 \\
\hline \multicolumn{14}{|l|}{ Study design } \\
\hline Retrospective & 12 & 1.00 & 0.87 to 1.15 & 0.970 & 73.6 & 1.07 & 0.86 to 1.33 & 0.540 & 52.8 & 1.05 & 0.87 to 1.28 & 0.599 & 63.9 \\
\hline Prospective & 4 & 1.21 & 1.01 to 1.25 & 0.038 & 46.9 & 1.27 & 1.02 to 1.59 & 0.037 & 49.7 & 1.28 & 1.07 to 1.54 & 0.007 & 47.3 \\
\hline \multicolumn{14}{|l|}{ Matched status } \\
\hline YES & 11 & 1.01 & 0.88 to 1.16 & 0.880 & 76.4 & 1.11 & 0.90 to 1.37 & 0.331 & 57.8 & 1.11 & 0.93 to 1.33 & 0.241 & 64.8 \\
\hline NR & 5 & 1.11 & 0.99 to 1.24 & 0.072 & 25.0 & 1.23 & 0.96 to 1.57 & 0.104 & 32.3 & 1.17 & 0.91 to 1.52 & 0.220 & 57.8 \\
\hline \multicolumn{14}{|l|}{ Sample size } \\
\hline$<500$ subjects & 6 & 0.85 & 0.65 to 1.12 & 0.251 & 7.9 & 0.85 & 0.58 to 1.24 & 0.407 & 44.2 & 0.84 & 0.62 to 1.15 & 0.285 & 48.2 \\
\hline$\geq 500$ subjects & 10 & 1.12 & 1.04 to 1.21 & 0.004 & 46.2 & 1.25 & 1.07 to 1.46 & 0.005 & 44.0 & 1.25 & 1.09 to 1.43 & 0.001 & 51.8 \\
\hline
\end{tabular}

Notes: OR, odds ratio; $95 \% \mathrm{CI}, 95 \%$ confidence interval; NR, not reported.

data from published papers were abstracted, and it could yield further insights if individual participant data were analyzed. Second, we were unable to glean various potential confounders (smoking, dyslipidemia, hypertension and diabetes) from all eligible studies, and only five studies provided complete confounding data. We adopted meta-regression analyses in an attempt to account for this limitation, while no significance was identified. Third, only one promoter polymorphism, C-629A in CETP was meta-analyzed, which is clearly not sufficient to support the contributory role of CETP in the pathogenesis of CHD and lipid regulation, as other polymorphisms in or flanking CETP might synergize or antagonize the impact of C-629A. Fourth, the association of CETP C-629A polymorphism with CHD risk and circulating lipid changes is not based on the same dataset due to limited number of qualified studies. Fifth, as with most meta-analyses, publication bias might be possible because only published articles were retrieved and the 'grey' literature (articles in languages other than English) was not reviewed. In view of these limitations, the jury must refrain from jumping at a conclusion until further verification of our findings in large, long-term, welldesigned prospective studies.

Taken together, our meta-analytic findings demonstrate that the $-629 \mathrm{C}$ allele was significantly associated with an increased risk of CHD in Caucasians, and this association may be mediated by its phenotypic regulation on circulating CETP and HDL-C. Although further investigations are required to elucidate the molecular mechanisms of CETP C-629A polymorphism underlying $\mathrm{CHD}$, future studies on the relationship between CETP genetic defects and CHD susceptibility need to focus on gene-to-environment interactions, especially on the impact of the $-629 \mathrm{C}$ allele on circulating lipid changes. 
Allelic model

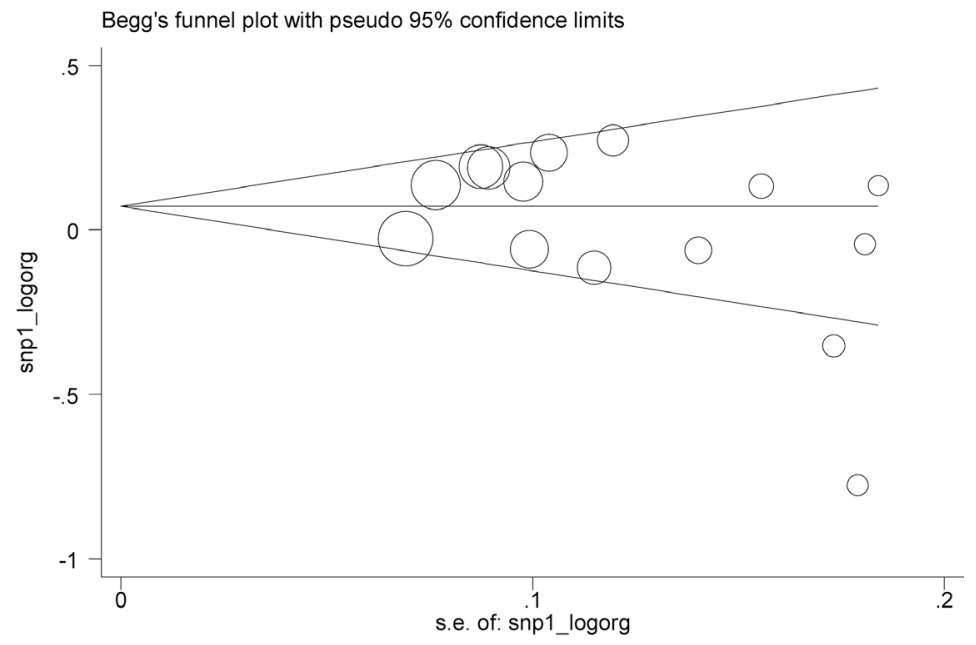

\section{Genotypic model}

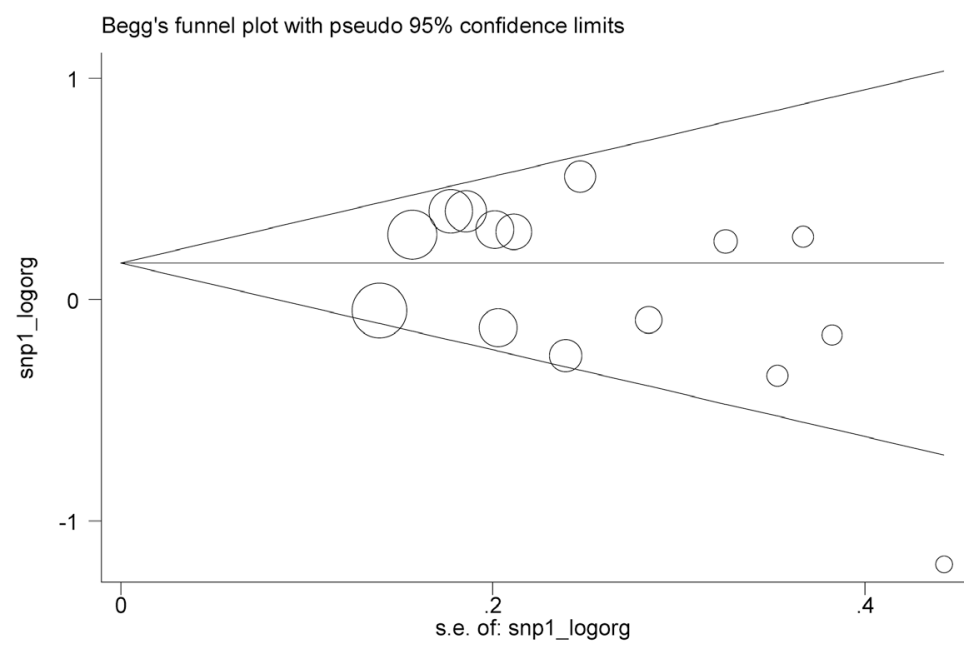

\section{Dominant model}

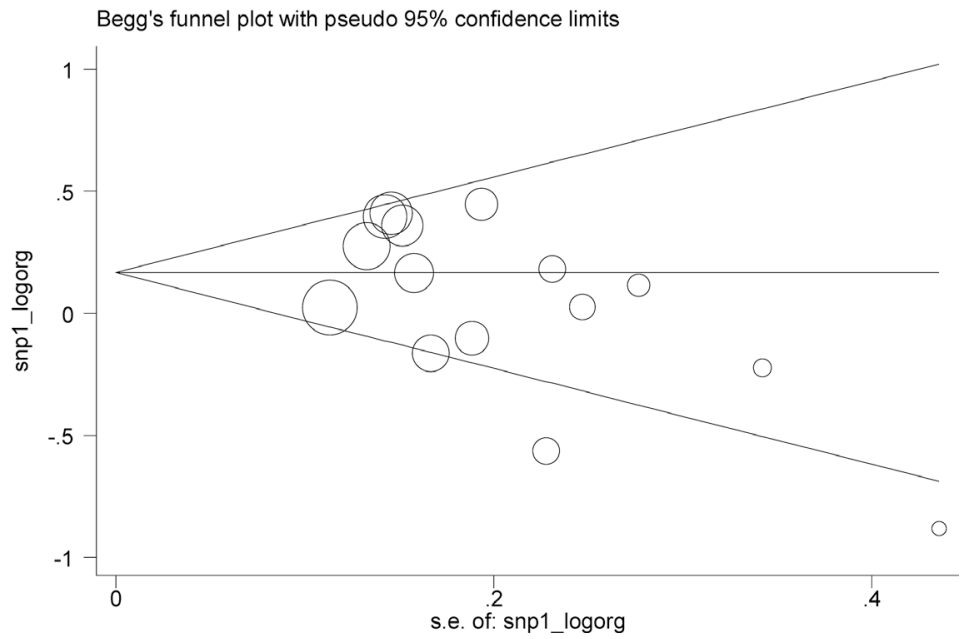

Figure 2: The Begg's funnel plots for the association of CETP C-629A polymorphism with CHD risk under three genetic models. Each hollow circle in Begg's funnel plots denotes each study, and the size of circle is positively proportional to the sample size of each study. 
Table 3: Overall analyses of CETP gene C-629A with circulating lipids under both genotypic and dominant models

\begin{tabular}{|c|c|c|c|c|c|c|}
\hline Lipids & Genetic models & Studies & WMD & $95 \%$ CI & $P$ & $I^{2}(\%)$ \\
\hline \multirow{2}{*}{ CETP } & Genotypic & 4 & 0.70 & 0.30 to 1.10 & 0.001 & 83.1 \\
\hline & Dominant & 4 & 0.45 & 0.25 to 0.65 & $<0.001$ & 69.3 \\
\hline \multirow{2}{*}{ Triglycerides } & Genotypic & 9 & -2.11 & -13.30 to 9.07 & 0.711 & 12.9 \\
\hline & Dominant & 9 & -0.77 & -12.41 to 10.86 & 0.896 & 42.3 \\
\hline \multirow{2}{*}{ HDL-C } & Genotypic & 16 & -4.36 & -7.20 to -1.51 & 0.003 & 84.2 \\
\hline & Dominant & 16 & -3.65 & -5.59 to -1.70 & $<0.001$ & 78.7 \\
\hline \multirow{2}{*}{ LDL-C } & Genotypic & 9 & 9.60 & -0.60 to 19.80 & 0.065 & 74.8 \\
\hline & Dominant & 9 & 7.03 & -0.62 to 14.67 & 0.072 & 69.6 \\
\hline \multirow{2}{*}{ Apo-AI } & Genotypic & 3 & -0.75 & -6.60 to 5.10 & 0.800 & 0.0 \\
\hline & Dominant & 3 & -3.66 & -7.76 to 0.43 & 0.079 & 0.0 \\
\hline \multirow{2}{*}{ Apo-B } & Genotypic & 3 & 4.77 & -3.34 to 12.87 & 0.249 & 0.0 \\
\hline & Dominant & 3 & 4.91 & -1.10 to 10.91 & 0.109 & 0.0 \\
\hline
\end{tabular}

Notes: WMD, weighted mean difference; 95\% CI, 95\% confidence interval.

\section{MATERIALS AND METHODS}

This meta-analysis of observational studies was conducted according to the PRISMA (Preferred Reporting Items for Systematic Reviews and Meta-analyses) statement [35]. All observational studies included were reported to obtain ethical approvals from the Ethics Committees of local institutions or departments.

\section{Search strategy}

PubMed/Medline, Embase, Scopus and Google Scholar electronic resources were searched on July 21, 2016 to seek articles of potential relevance, by using subject headings ('cholesteryl ester transfer protein' or 'cholesterol ester transfer protein' or 'CETP' and 'coronary heart disease' or 'isch[a]emic heart disease' or 'myocardial infarction' or 'atherosclerosis' or 'arteriosclerosis' or 'coronary artery disease' or 'coronary disease') and ('polymorphism' or 'variant' or 'variation' or 'mutation' or 'SNP'). The bibliographies of retrieved articles were also reviewed for articles that might be missed.

\section{Eligibility assessment}

The eligibility of each article was justified by two investigators (Shouwei Lin and Rong Lin) by reading the title and abstract, and if necessary the full text. Meanwhile, the period and location, if available, for study subjects collected were recorded to judge whether there were multiple publications from the same study. If so, the publication with a larger sample size was retained.

To be more specific, three inclusion criteria were proposed: (1) only English-language publications were considered; (2) the association of CETP C-629A polymorphism with CHD risk or circulating lipid changes was evaluated; (3) the absolute counts of C-629A genotypes between CHD patients and controls or the circulating lipid concentrations across C-629A genotypes were provided. In addition, articles were not taken into account if they were conference abstracts/posters, case reports, editorials and narrative/systematic reviews.

\section{Data extraction}

The same two investigators (Shouwei Lin and Rong Lin) independently extracted data from each qualified article according to a jointly formulated protocol, including first author's surname, publication year, ethnicity, diagnostic criteria of CHD including coronary stenosis or myocardial infarction, study design (retrospective or prospective design), source of controls (hospitals or populations), matched situation, sample size, absolute genotype counts of CETP C-629A polymorphism 
between CHD patients and controls or mean (standard deviation) concentrations of circulating CETP, triglycerides, HDL-C, LDL-C, Apo-AI and Apo-B across C-629A genotypes, as well as age, male gender, body mass index (BMI), smoking, dyslipidemia, hypertension, diabetes, circulating triglycerides, total cholesterol, HDL-C, LDL-C, CETP, Apo-AI and Apo-B, if available, between CHD patients and controls. For the sake of consistency, circulating triglycerides, total cholesterol, HDL-C, LDL-C, Apo-AI and Apo-B were expressed in $\mathrm{mg} / \mathrm{dL}$ and CETP in $\mu \mathrm{g} / \mathrm{mL}$.

\section{Statistical analysis}

Unadjusted odds ratio (OR) and weighted mean difference (WMD), along with $95 \%$ confidence interval ( $95 \% \mathrm{CI})$ were calculated by using a random-effects model with the DerSimonian \& Laird method to pool individual effect-size estimates under all circumstances. The magnitude of statistical heterogeneity across studies was represented by the inconsistency index $\left(I^{2}\right)$ statistic (range: $0 \%$ to $100 \%$ ). Statistical heterogeneity was reported to be significant if the $I^{2}$ statistic is over $50 \%$, which is a generally accepted cutoff value [36].

To explore the possible causes of clinical heterogeneity, grouping all qualified studies by gender, ethnicity, CHD subtype, control source, study design, matched status and sample size was conducted, separately. In addition, clinical heterogeneity was explored by metaregression analyses that incorporated all available discrete and continuous variables under study.

To see how effect estimates have shifted over time, cumulative analyses were performed in time sequence with each sub-analysis incorporating one additional study. To examine the robustness of overall estimates, influential analyses were undertaken by excluding each study from the analysis to seek its impact on the overall findings.

The assessment of publication bias was made by the Begg's funnel plots and Egger's asymmetry tests. The Egger's test can inspect funnel plot asymmetry by determining whether the intercept deviates significantly from zero when regressing the standardized effect estimates against their precision [37]. $P<0.10$ was chosen for the significance of Egger's tests. Data were statistically analyzed by the STATA software version 14.0 for Windows 10.0 (StataCorp, College Station, TX, USA).

\section{CONFLICTS OF INTEREST}

The authors declare that they have no competing interests.

\section{Authors' contributions}

Shouwei Lin conceived and designed this study; Shouwei Lin and Rong Lin performed literature search and data abstraction; Shouwei Lin performed statistical analyses; Shouwei Lin and Ruozhu Dai prepared draft materials; Shouwei Lin and Rong Lin wrote and revised the manuscript. All authors read and approved the final manuscript prior to submission.

\section{REFERENCES}

1. Oikawa S, Yokoyama M, Origasa H, Matsuzaki M, Matsuzawa Y, Saito Y, Ishikawa Y, Sasaki J, Hishida H, Itakura H, Kita T, Kitabatake A, Nakaya N, et al. Suppressive effect of EPA on the incidence of coronary events in hypercholesterolemia with impaired glucose metabolism: Sub-analysis of the Japan EPA Lipid Intervention Study (JELIS). Atherosclerosis. 2009; 206:535-539.

2. Li S, Zhao X, Zhang Y, Zhu CG, Guo YL, Wu NQ, Xu RX, Qing P, Gao Y, Sun J, Liu G, Dong Q, Li JJ. Novel circulating lipid measurements for current dyslipidemias in non-treated patients undergoing coronary angiography: PCSK9, apoC3 and sdLDL-C. Oncotarget. 2016 Oct 4. doi: 10.18632/oncotarget.12471. [Epub ahead of print].

3. Kuivenhoven JA, Jukema JW, Zwinderman AH, de Knijff P, McPherson R, Bruschke AV, Lie KI, Kastelein JJ. The role of a common variant of the cholesteryl ester transfer protein gene in the progression of coronary atherosclerosis. The Regression Growth Evaluation Statin Study Group. N Engl J Med. 1998; 338:86-93.

4. Thompson A, Di Angelantonio E, Sarwar N, Erqou S, Saleheen D, Dullaart RP, Keavney B, Ye Z, Danesh J. Association of cholesteryl ester transfer protein genotypes with CETP mass and activity, lipid levels, and coronary risk. JAMA. 2008; 299:2777-2788.

5. Dachet C, Poirier O, Cambien F, Chapman J, Rouis M. New functional promoter polymorphism, CETP/-629, in cholesteryl ester transfer protein (CETP) gene related to CETP mass and high density lipoprotein cholesterol levels: role of $\mathrm{Sp} 1 / \mathrm{Sp} 3$ in transcriptional regulation. Arterioscler Thromb Vasc Biol. 2000; 20:507-515.

6. Le Goff W, Guerin M, Petit L, Chapman MJ, Thillet J. Regulation of human CETP gene expression: role of SP1 and SP3 transcription factors at promoter sites -690, -629, and -37. J Lipid Res. 2003; 44:1322-1331.

7. Eiriksdottir G, Bolla MK, Thorsson B, Sigurdsson G, Humphries SE, Gudnason V. The $-629 \mathrm{C}>\mathrm{A}$ polymorphism in the CETP gene does not explain the association of TaqIB polymorphism with risk and age of myocardial infarction in Icelandic men. Atherosclerosis. 2001; 159:187-192.

8. Freeman DJ, Samani NJ, Wilson V, McMahon AD, Braund PS, Cheng S, Caslake MJ, Packard CJ, Gaffney D. A polymorphism of the cholesteryl ester transfer protein gene predicts cardiovascular events in non-smokers in the West of Scotland Coronary Prevention Study. Eur Heart J. 2003; 24:1833-1842.

9. Tobin MD, Braund PS, Burton PR, Thompson JR, Steeds R, Channer K, Cheng S, Lindpaintner K, Samani NJ. 
Genotypes and haplotypes predisposing to myocardial infarction: a multilocus case-control study. Eur Heart J. 2004; 25:459-467.

10. Zheng KQ, Zhang SZ, He Y, Zhang L, Zhang KL, Huang DJ, Sun Y. Association between cholesteryl ester transfer protein gene polymorphisms and variations in lipid levels in patients with coronary heart disease. Chin Med J (Engl). 2004; 117:1288-1292.

11. Zee RY, Cook NR, Cheng S, Erlich HA, Lindpaintner K, Ridker PM. Multi-locus candidate gene polymorphisms and risk of myocardial infarction: a population-based, prospective genetic analysis. J Thromb Haemost. 2006; 4:341-348.

12. Meiner V, Friedlander Y, Milo H, Sharon N, Ben-Avi L, Shpitzen S, Leitersdorf E, Siscovick DS, Schwartz SM. Cholesteryl ester transfer protein (CETP) genetic variation and early onset of non-fatal myocardial infarction. Ann Hum Genet. 2008; 72:732-741.

13. Ghatreh Samani K, Noori $M$, Rohbani Nobar $M$, Hashemzadeh Chaleshtori M, Farrokhi E, Darabi Amin M. $\mathrm{I} 405 \mathrm{~V}$ and $-629 \mathrm{C} / \mathrm{A}$ polymorphisms of the cholesteryl ester transfer protein gene in patients with coronary artery disease. Iran Biomed J. 2009; 13:103-108.

14. Padmaja N, Kumar RM, Balachander J, Adithan C. Cholesteryl ester transfer protein TaqIB, $-629 \mathrm{C}>\mathrm{A}$ and I405V polymorphisms and risk of coronary heart disease in an Indian population. Clin Chim Acta. 2009; 402:139-145.

15. Poduri A, Khullar M, Bahl A, Sharma YP, Talwar KK. A combination of proatherogenic single-nucleotide polymorphisms is associated with increased risk of coronary artery disease and myocardial infarction in Asian Indians. DNA Cell Biol. 2009; 28:451-460.

16. Tanrikulu S, Ademoglu E, Gurdol F, Mutlu-Turkoglu U, Bilge AK, Nisanci Y. Association of cholesteryl ester transfer protein $-629 \mathrm{C}>$ A polymorphism with high-density lipoprotein cholesterol levels in coronary artery disease patients. Cell Biochem Funct. 2009; 27:452-457.

17. Lu Y, Tayebi N, Li H, Saha N, Yang H, Heng CK. Association of CETP Taq1B and -629C > A polymorphisms with coronary artery disease and lipid levels in the multi-ethnic Singaporean population. Lipids Health Dis. 2013; 12:85.

18. Wang J, Wang LJ, Zhong Y, Gu P, Shao JQ, Jiang SS, Gong JB. CETP gene polymorphisms and risk of coronary atherosclerosis in a Chinese population. Lipids Health Dis. 2013; 12:176.

19. Kakko S, Tamminen M, Paivansalo M, Kauma H, Rantala AO, Lilja M, Reunanen A, Kesaniemi YA, Savolainen MJ. Variation at the cholesteryl ester transfer protein gene in relation to plasma high density lipoproteins cholesterol levels and carotid intima-media thickness. Eur J Clin Invest. 2001; 31:593-602.

20. Yilmaz H, Isbir T, Agachan B, Karaali ZE. Effects of cholesterol ester transfer protein Taq1B gene polymorphism on serum lipoprotein levels in Turkish coronary artery disease patients. Cell Biochem Funct. 2005; 23:23-28.
21. Tsai MY, Johnson C, Kao WH, Sharrett AR, Arends VL, Kronmal R, Jenny NS, Jacobs DR, Jr., Arnett D, O'Leary D, Post W. Cholesteryl ester transfer protein genetic polymorphisms, HDL cholesterol, and subclinical cardiovascular disease in the Multi-Ethnic Study of Atherosclerosis. Atherosclerosis. 2008; 200:359-367.

22. Ridker PM, Pare G, Parker AN, Zee RY, Miletich JP, Chasman DI. Polymorphism in the CETP gene region, HDL cholesterol, and risk of future myocardial infarction: Genomewide analysis among 18245 initially healthy women from the Women's Genome Health Study. Circ Cardiovasc Genet. 2009; 2:26-33.

23. Wu W, Wu Y, Wang M, Zhang D. Meta-analysis of the association between the XRCC1 gene R399Q polymorphism and colorectal cancer: an update. Int $\mathrm{J}$ Colorectal Dis. 2013; 28:1453-1454.

24. Gu GL, Xu XL, Yang QY, Zeng RL. Effect of CETP polymorphism on atorvastatin lipid-regulating effect and clinical prognosis of patients with coronary heart disease. Med Sci Monit. 2014; 20:2824-2829.

25. Niu W, Qi Y. Circulating cholesteryl ester transfer protein and coronary heart disease: mendelian randomization metaanalysis. Circ Cardiovasc Genet. 2015; 8:114-121.

26. Smith GD, Ebrahim S. Mendelian randomization: prospects, potentials, and limitations. Int J Epidemiol. 2004; 33:30-42.

27. Yu K, Zhang J, Dou C, Gu S, Xie Y, Mao Y, Ji C. Methionine synthase A2756G polymorphism and cancer risk: a meta-analysis. Eur J Hum Genet. 2010; 18:370-378.

28. Nagano M, Yamashita S, Hirano K, Takano M, Maruyama T, Ishihara M, Sagehashi Y, Kujiraoka T, Tanaka K, Hattori H, Sakai N, Nakajima N, Egashira T, Matsuzawa Y. Molecular mechanisms of cholesteryl ester transfer protein deficiency in Japanese. J Atheroscler Thromb. 2004; 11:110-121.

29. Qi Y, Fan J, Liu J, Wang W, Wang M, Sun J, Xie W, Zhao F, Li Y, Zhao D. Cholesterol-overloaded HDL particles are independently associated with progression of carotid atherosclerosis in a cardiovascular disease-free population: a community-based cohort study. J Am Coll Cardiol. 2015; 65:355-363.

30. Gauthier A, Lau P, Zha X, Milne R, McPherson R. Cholesteryl ester transfer protein directly mediates selective uptake of high density lipoprotein cholesteryl esters by the liver. Arterioscler Thromb Vasc Biol. 2005; 25:2177-2184.

31. Berard AM, Foger B, Remaley A, Shamburek R, Vaisman BL, Talley G, Paigen B, Hoyt RF, Jr., Marcovina S, Brewer HB, Jr. and Santamarina-Fojo S. High plasma HDL concentrations associated with enhanced atherosclerosis in transgenic mice overexpressing lecithin-cholesteryl acyltransferase. Nat Med. 1997; 3:744-749.

32. Collet X, Tall AR, Serajuddin H, Guendouzi K, Royer L, Oliveira H, Barbaras R, Jiang XC, Francone OL. Remodeling of HDL by CETP in vivo and by CETP and hepatic lipase in vitro results in enhanced uptake of HDL CE by cells expressing scavenger receptor B-I. J Lipid Res. 1999; 40:1185-1193. 
33. Rashedi N, Brennan D, Kastelein JJ, Nissen SE, S. N. Impact of cholesteryl ester transfer protein inhibition on nuclear magnetic resonance derived lipoprotein particle parameters Atherosclerosis Supplements 2011; 12:48-48.

34. Jafri H, Alsheikh-Ali AA, Mooney P, Kimmelstiel CD, Karas RH, Kuvin JT. Extended-release niacin reduces LDL particle number without changing total LDL cholesterol in patients with stable CAD. J Clin Lipidol. 2009; 3:45-50.

35. Moher D, Liberati A, Tetzlaff J, Altman DG. Preferred reporting items for systematic reviews and meta-analyses: the PRISMA statement. Ann Intern Med. 2009; 151:264-269, W264.
36. Higgins JP, Thompson SG, Deeks JJ, Altman DG. Measuring inconsistency in meta-analyses. BMJ. 2003; 327:557-560.

37. Egger M, Davey Smith G, Schneider M, Minder C. Bias in meta-analysis detected by a simple, graphical test. BMJ. 1997; 315:629-634. 